\title{
Poisson Quasi-Nijenhuis Manifolds
}

\author{
Mathieu StiÉnon * \\ Departement Mathematik \\ E.T.H. Zürich \\ Rämistrasse 101 \\ 8092 Zürich, Switzerland \\ mathieu.stienon@math.ethz.ch
}

\author{
PING $\mathrm{XU}^{\dagger}$ \\ Department of Mathematics \\ Pennsylvania State University \\ University Park, PA 16802 \\ USA \\ ping@math . psu .edu
}

\begin{abstract}
We introduce the notion of Poisson quasi-Nijenhuis manifolds generalizing the PoissonNijenhuis manifolds of Magri-Morosi. We also investigate the integration problem of Poisson quasi-Nijenhuis manifolds. In particular, we prove that, under some topological assumption, Poisson (quasi)-Nijenhuis manifolds are in one-one correspondence with symplectic (quasi)-Nijenhuis groupoids. As an application, we study generalized complex structures in terms of Poisson quasi-Nijenhuis manifolds. We prove that a generalized complex manifold corresponds to a special class of Poisson quasi-Nijenhuis structures. As a consequence, we show that a generalized complex structure integrates to a symplectic quasi-Nijenhuis groupoid recovering a theorem of Crainic.
\end{abstract}

\section{Contents}

1 Introduction $\quad 2$

2 Preliminaries $\quad 4$

3 Poisson quasi-Nijenhuis manifolds $\quad 6$

4 Universal lifting theorem $\quad 10$

5 Symplectic Nijenhuis groupoids $\quad 12$

6 Symplectic quasi-Nijenhuis groupoids $\quad 13$

7 Generalized complex structures $\quad 14$

${ }^{*}$ Francqui fellow of the Belgian American Educational Foundation
${ }^{\dagger}$ Research supported by NSF grant DMS03-06665 and NSA grant 03G-142. 


\section{Introduction}

Poisson Nijenhuis structures were introduced by Magri and Morosi [16, 18] in their study of bi-Hamiltonian systems, and intensively studied by many authors [12, 21]. Recall that a Poisson Nijenhuis manifold consists of a triple $(M, \pi, N)$, where $M$ is a manifold endowed with a Poisson bivector field $\pi$, and a $(1,1)$-tensor $N$ whose Nijenhuis torsion vanishes, i.e.

$$
[N X, N Y]-N([N X, Y]+[X, N Y]-N[X, Y])=0, \quad \forall X, Y \in \mathfrak{X}(M),
$$

together with some compatibility condition between $\pi$ and $N$. Poisson Nijenhuis structures are very important in the study of integrable systems since they produce bi-Hamiltonian systems $[16,12]$.

As observed by Kosmann-Schwarzbach [11], given a Poisson Nijenhuis manifold $(M, \pi, N)$, $\left(\left(T^{*} M\right)_{\pi},(T M)_{N}\right)$ constitutes a Lie bialgebroid, where $\left(T^{*} M\right)_{\pi}$ is equipped with the standard cotangent Lie algebroid structure induced by the Poisson tensor $\pi$ while $(T M)_{N}$ is the deformed Lie algebroid on $T M$ induced by the Nijenhuis endomorphism $N$. Indeed it is proved in [11] that the Lie bialgebroid condition on $\left(\left(T^{*} M\right)_{\pi},(T M)_{N}\right)$ is equivalent to the triple $(M, \pi, N)$ being Poisson Nijenhuis.

The main goal of the present paper is to introduce the notion of Poisson quasi-Nijenhuis structures. By definition, a Poisson quasi-Nijenhuis manifold is a quadruple $(M, \pi, N, \phi)$, where $M$ is manifold endowed with a Poisson bivector field $\pi$, a $(1,1)$-tensor $N$ and a closed 3 -form $\phi$ such that $\pi$ and $N$ are compatible (in the usual Poisson-Nijenhuis sense) and

$$
[N X, N Y]-N([N X, Y]+[X, N Y]-N[X, Y])=\pi^{\sharp}\left(i_{X \wedge Y} \phi\right), \quad \forall X, Y \in \mathfrak{X}(M) .
$$

Recall that Lie bialgebroids are pairs of transverse Dirac structures in a Courant algebroid [13]. When one of the two maximal isotropic direct summands fails to be Courant involutive, this becomes a quasi-Lie bialgebroid [20,19]. Alternatively, a quasi-Lie bialgebroid is equivalent to the following data: a Lie algebroid $A$ together with a degree 1 derivation $\delta$ of the associated Gerstenhaber algebra $\left(\Gamma\left(\wedge^{\bullet} A\right), \wedge,[\cdot, \cdot]\right)$ such that $\delta^{2}=[\phi, \cdot]$ and $\delta \phi=0$ for some $\phi \in \Gamma\left(\wedge^{3} A\right)$ [9]. We prove

Theorem A. Given $(M, \pi, N, \phi)$, the following are equivalent

- $(M, \pi, N, \phi)$ is a Poisson quasi-Nijenhuis manifold;

- $\left(\left(T^{*} M\right)_{\pi},(T M)_{N}, \phi\right)$ is a quasi-Lie bialgebroid.

It is well known that the global object corresponding to a Poisson manifold is a symplectic groupoid $[2,22]$. It is natural to ask what is the global object integrating a Poisson Nijenhuis manifold. We prove

Theorem B. The base manifold of a symplectic Nijenhuis groupoid is a Poisson Nijenhuis manifold. Moreover, there is a one-one correspondence between $t$-connected and $t$-simply connected symplectic Nijenhuis groupoids $(\Gamma \rightrightarrows M, \widetilde{\omega}, \widetilde{N})$ and integrable Poisson Nijenhuis manifolds $(M, \pi, N)$.

By a symplectic Nijenhuis groupoid, we mean a symplectic groupoid $(\Gamma \rightrightarrows M, \widetilde{\omega})$ equipped with a multiplicative $(1,1)$-tensor $\widetilde{N}: T \Gamma \rightarrow T \Gamma$ such that $(\Gamma, \widetilde{\omega}, \widetilde{N})$ is a symplectic Nijenhuis structure. The main idea of the proof of Theorem B can be outlined as follows. One proves that Poisson Nijenhuis structures on a manifold $M$ are in one-one correspondence with Lie 
bialgebroids $\left(\left(T^{*} M\right)_{\pi}, \delta\right)$ satisfying the condition that $[\delta, d]=0$, where $d$ is the de Rham differential on $M$. The latter are the infinitesimal of symplectic Nijenhuis groupoids, as can be shown using the universal lifting theorem [9].

The same method can be used to prove an analogous result for Poisson quasi-Nijenhuis manifolds.

Theorem C. The base manifold of a symplectic quasi-Nijenhuis groupoid is a Poisson quasiNijenhuis manifold. Moreover there is a one-one correspondence between t-connected and $t$ simply connected symplectic quasi-Nijenhuis groupoids $\left(\Gamma \rightrightarrows M, \widetilde{\omega}, \widetilde{N}, t^{*} \phi-s^{*} \phi\right)$ and integrable Poisson quasi-Nijenhuis manifolds $(M, \pi, N, \phi)$.

A symplectic quasi-Nijenhuis groupoid is a symplectic groupoid $(\Gamma \rightrightarrows M, \widetilde{\omega}$ ) equipped with a multiplicative $(1,1)$-tensor $\widetilde{N}: T \Gamma \rightarrow T \Gamma$ and a closed 3-form $\phi \in \Omega^{3}(M)$ such that $\left(\Gamma, \widetilde{\omega}, \widetilde{N}, t^{*} \phi-s^{*} \phi\right)$ is a symplectic quasi-Nijenhuis structure.

As an application, we study generalized complex structures in terms of Poisson quasi-Nijenhuis structures. The notion of generalized complex structures was introduced by Hitchin [8] and studied by Gualtieri [7] motivated by the study of mirror symmetry. It comprises both symplectic and complex structures as extreme cases. We show that on a generalized complex manifold $(M, J)$, where

$$
J=\left(\begin{array}{cc}
N & \pi^{\sharp} \\
\sigma_{b} & -N^{*}
\end{array}\right)
$$

with $N^{2}+\pi^{\sharp} \sigma_{b}=-$ id, the building units $\pi, N$ and $\sigma$ of $J$ do exactly determine a Poisson quasi-Nijenhuis structure. Indeed, the endomorphism $N$ can be used to define a derivation $d_{N}$ of the Gerstenhaber algebra associated to the Lie algebroid $\left(T^{*} M\right)_{\pi}$. We prove

Theorem D. The following are equivalent

- $J$ is a generalized complex structure;

- $(M, \pi, N, d \sigma)$ is a Poisson quasi-Nijenhuis structure such that

$$
(T M)_{N} \oplus\left(T^{*} M\right)_{\pi} \stackrel{J}{\rightarrow} T M \oplus T^{*} M
$$

is a Courant algebroid isomorphism.

A similar result (in a different form) was already proved by Crainic using a direct argument [4].

Since a generalized complex structure corresponds to a quasi-Nijenhuis manifold according to Theorem D, as a consequence, we prove

Theorem E. Let $J$ be a generalized complex structure as given by $E q .(18)$, and $(\Gamma \rightrightarrows M, \widetilde{\omega})$ a t-connected and t-simply connected symplectic groupoid integrating $\left(T^{*} M\right)_{\pi}$. Then there is a multiplicative $(1,1)$-tensor $\widetilde{N}$ on $\Gamma$ such that $\left(\Gamma \rightrightarrows M, \widetilde{\omega}, \widetilde{N}, t^{*} d \sigma-s^{*} d \sigma\right)$ is a symplectic quasi-Nijenhuis groupoid.

This result, in a disguised form, was also proved by Crainic [4] using a different method. 
Notations We denote the bracket on the sections of a Courant algebroid by $\llbracket \cdot, \cdot \rrbracket$, except for the standard Courant bracket on $T M \oplus T^{*} M$, which is denoted by $(\cdot, \cdot)$. The Lie bracket of vector fields and its extension to polyvector fields (i.e. the Schouten bracket) are denoted by $[\cdot, \cdot]$. Any bundle map $B: T^{*} M \rightarrow T M$ induces a bracket on the space of 1 -forms (see Eq. (8)). It is denoted by $[\cdot, \cdot]_{B}$ as well as its extension to the space of differential forms of all degrees. Finally, if $\llbracket \cdot, \cdot \rrbracket$ is a bracket on the space of sections of a vector bundle $E$ of which $J$ is a bundle endomorphism, then its deformation by $J$ is denoted by $\llbracket \cdot, \cdot \rrbracket_{J}$ (see Eq. (19)).

Acknowledgments We would like to thank several institutions for their hospitality while work on this project was being done: Erwin Schroedinger International Institute for Mathematical Physics (Stiénon and $\mathrm{Xu}$ ), and Université Pierre et Marie Curie (Xu). Stiénon is grateful to the Émile Francqui and Belgian American Educational Foundations for supporting his stay at the Pennsylvania State University where this work was completed in 2005. We would also like to thank Marius Crainic and Jim Stasheff for many useful discussions, and the referee for helpful suggestions to improve the presentation of the paper.

\section{Preliminaries}

Definition 2.1 ([13]). A Courant algebroid is a triple consisting of

- a vector bundle $E \rightarrow M$ equipped with a non degenerate symmetric bilinear form $\langle\cdot, \cdot\rangle$,

- a skew-symmetric bracket $\llbracket \cdot, \cdot \rrbracket$ on $\Gamma(E)$, and

- a smooth bundle map $E \stackrel{\rho}{\rightarrow} M$ called the anchor, which induces a natural differential operator $\mathcal{D}: C^{\infty}(M) \rightarrow \Gamma(E)$ defined by

$$
\langle\mathcal{D} f, A\rangle=\frac{1}{2} \rho(A) f
$$

for all $f \in C^{\infty}(M)$ and $A \in \Gamma(E)$.

These structures must be compatible in the following sense: $\forall A, B, C \in \Gamma(E)$ and $\forall f, g \in$ $C^{\infty}(M)$

- $\rho(\llbracket A, B \rrbracket)=[\rho(A), \rho(B)]$,

- $\llbracket \llbracket A, B \rrbracket, C \rrbracket+\llbracket \llbracket B, C \rrbracket, A \rrbracket+\llbracket \llbracket C, A \rrbracket, B \rrbracket=\frac{1}{3} \mathcal{D}(\langle\llbracket A, B \rrbracket, C\rangle+\langle\llbracket B, C \rrbracket, A\rangle+\langle\llbracket C, A \rrbracket, B\rangle)$,

- $\llbracket A, f B \rrbracket=f \llbracket A, B \rrbracket+(\rho(A) f) B-\langle A, B\rangle \mathcal{D} f$,

- $\rho_{\circ} \mathcal{D}=0$, i.e. $\langle\mathcal{D} f, \mathcal{D} g\rangle=0$,

- $\rho(A)\langle B, C\rangle=\langle\llbracket A, B \rrbracket+\mathcal{D}\langle A, B\rangle, C\rangle+\langle B, \llbracket A, C \rrbracket+\mathcal{D}\langle A, C\rangle\rangle$.

Note that a Courant algebroid is not a Lie algebroid as the Jacobi identity is not satisfied.

Example 2.2 ([3]). The generalized tangent bundle $T M \oplus T^{*} M$ of a manifold $M$ is a Courant algebroid, where the anchor is the projection onto the first component and the pairing and bracket are given, respectively, by

$$
\begin{gathered}
\langle X+\xi, Y+\eta\rangle=\frac{1}{2}(\xi(Y)+\eta(X)), \\
(X+\xi, Y+\eta)=[X, Y]+\mathcal{L}_{X} \eta-\mathcal{L}_{Y} \xi+\frac{1}{2}(\xi(Y)-\eta(X)),
\end{gathered}
$$

$\forall X, Y \in \mathfrak{X}(M), \forall \xi, \eta \in \Omega^{1}(M)$. 
Definition 2.3. A Dirac structure is a smooth subbundle $L$ of a Courant algebroid $E$, which is maximal isotropic with respect to $\langle\cdot, \cdot\rangle$ and whose space of sections $\Gamma(L)$ is closed under $\llbracket \cdot, \cdot \rrbracket$. It is thus naturally a Lie algebroid.

It is well-known [23] that a Lie algebroid $\left(A,[\cdot, \cdot]_{A}, \rho_{A}\right)$ gives rise to a Gerstenhaber algebra $\left(\Gamma\left(\wedge^{\bullet} A\right), \wedge,[\cdot, \cdot]_{A}\right)$, and a degree 1 derivation $\delta_{A}$ of the graded commutative algebra $\left(\Gamma\left(\wedge^{\bullet} A^{*}\right), \wedge\right)$ such that $\left(\delta_{A}\right)^{2}=0$. Here $\delta_{A}$ is given by

$$
\begin{aligned}
\left(\delta_{A} \alpha\right)\left(X_{0}, X_{1}, \cdots, X_{n}\right)=\sum_{i=0}^{n}( & -1)^{i}\left(\rho_{A} X_{i}\right) \alpha\left(X_{0}, \cdots, \widehat{X}_{i}, \cdots, X_{n}\right) \\
& +\sum_{i<j}(-1)^{i+j} \alpha\left(\left[X_{i}, X_{j}\right]_{A}, X_{0}, \cdots, \widehat{X}_{i}, \cdots, \widehat{X}_{j}, \cdots, X_{n}\right) .
\end{aligned}
$$

A Lie bialgebroid $[15,14]$ is a pair of Lie algebroid structures on $A$ and its dual $A^{*}$ such that $\delta_{A^{*}}$ is a derivation of the Gerstenhaber algebra $\left(\Gamma\left(\wedge^{\bullet} A\right), \wedge,[\cdot, \cdot]_{A}\right)$ or, equivalently, such that $\delta_{A}$ is a derivation of the Gerstenhaber algebra $\left(\Gamma\left(\wedge^{\bullet} A^{*}\right), \wedge,[\cdot, \cdot]_{A^{*}}\right)$. Since the bracket $[\cdot, \cdot]_{A^{*}}$ can be recovered from the derivation $\delta_{A^{*}}$, one is led to the following alternative definition.

Definition 2.4. A Lie bialgebroid is a pair $(A, \delta)$ consisting of a Lie algebroid $\left(A,[\cdot, \cdot]_{A}, \rho_{A}\right)$ and a degree 1 derivation $\delta$ of the Gerstenhaber algebra $\left(\Gamma(\wedge \cdot A), \wedge,[\cdot, \cdot]_{A}\right)$ such that $\delta^{2}=0$.

More generally, we can speak about quasi-Lie bialgebroids [20, 9].

Definition 2.5 ([9]). A quasi Lie bialgebroid is a triple $(A, \delta, \phi)$ consisting of a Lie algebroid $A$, a degree 1 derivation $\delta$ of the Gerstenhaber algebra $\left(\Gamma\left(\wedge^{\bullet} A\right), \wedge,[\cdot, \cdot]_{A}\right)$ and an element $\phi \in \Gamma\left(\wedge^{3} A\right)$ such that $\delta^{2}=[\phi, \cdot]_{A}$ and $\delta \phi=0$.

The link between Courant, Lie bi- and quasi Lie bialgebroids is given by the following

Theorem 2.6 ([13, 20, 19]). (i) There is a 1-1 correspondence between Lie bialgebroids and pairs of transversal Dirac structures in a Courant algebroid.

(ii) There is a 1-1 correspondence between quasi Lie bialgebroids and Dirac structures with transversal isotropic complements in a Courant algebroid.

Proof. The proof of (i) can be found in [13], and (ii) was proved in [20, 19]. Below we give an explicit formula describing such a correspondence, which will be needed later.

Let $(A, \delta, \phi)$ be a quasi Lie bialgebroid. Let $\rho_{A *}: A^{*} \rightarrow T M$ be the bundle map given by

$$
\rho_{A^{*}}(\xi)(f)=\xi(\delta f), \quad \forall \xi \in A^{*}, \forall f \in C^{\infty}(M) .
$$

Introduce a bracket on $\Gamma\left(A^{*}\right)$ by

$$
[\xi, \eta]_{A^{*}}(X)=\left(\rho_{A^{*}} \xi\right)(\eta X)-\left(\rho_{A^{*}} \eta\right)(\xi X)-(\delta X)(\xi, \eta)
$$

Note that $\left(A^{*}, \rho_{A^{*}},[\cdot, \cdot]_{A *}\right)$ is in general not a Lie algebroid. Let $E=A^{*} \oplus A$ and $\rho: E \rightarrow T M$ be the bundle map

$$
\rho(\xi+X)=\rho_{A^{*}}(\xi)+\rho_{A}(X) .
$$

Define a non-degenerate symmetric pairing on $E$ by

$$
\langle\xi+X, \eta+Y\rangle=\frac{1}{2}(\xi(Y)+\eta(X)),
$$


and a bracket $\llbracket \cdot, \cdot \rrbracket$ on $\Gamma(E)$ by

$$
\begin{gathered}
\llbracket X, Y \rrbracket=[X, Y]_{A}, \\
\llbracket \xi, \eta \rrbracket=[\xi, \eta]_{A^{*}}+\phi(\xi, \eta, \cdot), \\
\llbracket X, \xi \rrbracket=\left(i_{X} \delta_{A^{*}} \xi+\frac{1}{2} \delta_{A^{*}}(\xi X)\right)-\left(i_{\xi} \delta_{A} X+\frac{1}{2} \delta_{A}(\xi X)\right),
\end{gathered}
$$

for all $X, Y \in \Gamma(A)$ and $\xi, \eta \in \Gamma\left(A^{*}\right)$. Here $\delta_{A *}: \Gamma\left(\wedge^{\bullet} A^{*}\right) \rightarrow \Gamma\left(\wedge^{\bullet+1} A^{*}\right)$ is the derivation given by Eq. (3). Then $(E,\langle\cdot, \cdot\rangle, \llbracket \cdot, \cdot \rrbracket, \rho)$ is a Courant algebroid.

Conversely, assume that $(E,\langle\cdot, \cdot\rangle, \llbracket \cdot, \cdot \rrbracket, \rho)$ is a Courant algebroid, and $A$ is a Dirac structure with an isotropic complement $B$. The duality pairing

$$
A \otimes B \rightarrow \mathbb{R}: X \otimes \xi \mapsto 2\langle\xi, X\rangle
$$

identifies $B$ with $A^{*}$. Let $\phi$ be the element in $\Gamma\left(\wedge^{3} A\right)$ defined by

$$
\phi(\xi, \eta, \zeta)=2\langle\llbracket \xi, \eta \rrbracket, \zeta\rangle, \quad \forall \xi, \eta, \zeta \in \Gamma(B),
$$

$\rho_{B}=\left.\rho\right|_{B}$ be the restriction of $\rho$ to $B$ and $[\cdot, \cdot]_{B}$ be the bracket on $\Gamma(B)$ such that

$$
\llbracket \xi, \eta \rrbracket-[\xi, \eta]_{B} \in \Gamma(A), \quad \forall \xi, \eta \in \Gamma(B) .
$$

Define a derivation $\delta: \Gamma\left(\wedge^{\bullet} A\right)\left(\cong \Gamma\left(\wedge^{\bullet} B^{*}\right)\right) \rightarrow \Gamma\left(\wedge^{\bullet+1} A\right)\left(\cong \Gamma\left(\wedge^{\bullet+1} B^{*}\right)\right.$ as in Eq. (3). The triple $(A, \delta, \phi)$ becomes a quasi Lie bialgebroid.

\section{Poisson quasi-Nijenhuis manifolds}

Let $M$ be a smooth manifold, $\pi$ a Poisson bivector field, and $N: T M \rightarrow T M$ a (1,1)-tensor.

Definition 3.1 ([11]). The bivector field $\pi$ and the tensor $N$ are said to be compatible [12] if

$$
N \circ \pi^{\sharp}=\pi^{\sharp} \circ N^{\mathrm{T}} \quad \text { and } \quad C_{\pi^{\sharp}}^{N}=0,
$$

where

$$
C_{\pi^{\sharp}}^{N}(\alpha, \beta):=[\alpha, \beta]_{N \pi^{\sharp}}-\left(\left[N^{\mathrm{T}} \alpha, \beta\right]_{\pi^{\sharp}}+\left[\alpha, N^{\mathrm{T}} \beta\right]_{\pi^{\sharp}}-N^{\mathrm{T}}[\alpha, \beta]_{\pi^{\sharp}}\right)
$$

and

$$
[\alpha, \beta]_{B}:=\mathcal{L}_{B \alpha}(\beta)-\mathcal{L}_{B \beta}(\alpha)-d(\beta(B \alpha))
$$

for all $\alpha, \beta \in \Omega^{1}(M)$ and any bundle map $B: T^{*} M \rightarrow T M$.

The (1,1)-tensor $N$ is said to have zero Nijenhuis torsion if

$$
[N X, N Y]-N([N X, Y]+[X, N Y]-N[X, Y])=0, \quad \forall X, Y \in \mathfrak{X}(M) .
$$

In [17], Magri and Morosi defined Poisson Nijenhuis manifolds as triples $(M, \pi, N)$ such that $\pi$ and $N$ are compatible and the Nijenhuis torsion of $N$ vanishes.

This definition is motivated by the following

Fact $3.2([12,21])$. Assume that $\pi \in \mathfrak{X}^{2}(M)$ is a Poisson tensor and $N: T M \rightarrow T M a$ $(1,1)$-tensor on $M$. The tensor $\pi_{N}$ defined by

$$
\pi_{N}(\alpha, \beta):=\beta\left(N \pi^{\sharp} \alpha\right), \quad \forall \alpha, \beta \in \Omega^{1}(M)
$$

is skew-symmetric if, and only if, $N_{\circ} \pi^{\sharp}=\pi_{\circ}^{\sharp} N^{T}$. In this case, we have 
(i) $\left[\pi, \pi_{N}\right]=0$ if $C_{\pi^{\sharp}}^{N}=0$, and the converse is true if $\pi$ is non-degenerate;

(ii) $\left[\pi_{N}, \pi_{N}\right]=0$ if, and only if, the Nijenhuis torsion of $N$ vanishes.

Hence, any Poisson Nijenhuis manifold $(M, \pi, N)$ is endowed with a bi-Hamiltonian structure $\left(\pi, \pi_{N}\right)$, i.e.

$$
[\pi, \pi]=0, \quad\left[\pi, \pi_{N}\right]=0, \quad\left[\pi_{N}, \pi_{N}\right]=0 .
$$

Similarly, one can define Poisson quasi-Nijenhuis manifolds.

Let $i_{N}$ be the degree 0 derivation of $\left(\Omega^{\bullet}(M), \wedge\right)$ defined by

$$
\left(i_{N} \alpha\right)\left(X_{1}, \cdots, X_{p}\right)=\sum_{i=1}^{p} \alpha\left(X_{1}, \cdots, N X_{i}, \cdots, X_{p}\right), \quad \forall \alpha \in \Omega^{p}(M) .
$$

Definition 3.3. A Poisson quasi-Nijenhuis manifold is a quadruple $(M, \pi, N, \phi)$, where $\pi \in$ $\mathfrak{X}^{2}(M)$ is a Poisson bivector field, $N: T M \rightarrow T M$ is a $(1,1)$-tensor compatible with $\pi$, and $\phi$ is a closed 3 -form on $M$ such that

$$
[N X, N Y]-N([N X, Y]+[X, N Y]-N[X, Y])=\pi^{\sharp}\left(i_{X \wedge Y} \phi\right), \quad \forall X, Y \in \mathfrak{X}(M)
$$

and $i_{N} \phi$ is closed.

It is well known that, on a Poisson manifold $(M, \pi)$, the bracket on $\Omega^{1}(M)$ associated to the bundle map $\pi^{\sharp}$ through Eq. (8) makes $T^{*} M$ into a Lie algebroid with anchor $\pi^{\sharp}: T^{*} M \rightarrow T M$. The usual cotangent bundle will be denoted by $\left(T^{*} M\right)_{\pi}$ when equipped with this Lie algebroid structure. More precisely, we have the following

Fact 3.4 ([2]). Let $\pi$ be a bivector field on $M$. Then $[\pi, \pi]=0$ if, and only if, $\left(T^{*} M\right)_{\pi}$ is a Lie algebroid.

On the other hand, defining a bracket $[\cdot, \cdot]_{N}$ on $\mathfrak{X}(M)$ by

$$
[X, Y]_{N}=[N X, Y]+[X, N Y]-N[X, Y], \quad \forall X, Y \in \mathfrak{X}(M)
$$

as in [11], and considering $N: T M \rightarrow T M$ as an anchor map, we obtain a degree 1 derivation $d_{N}$ of $(\Omega \bullet(M), \wedge)$ inspired by Eq. (3):

$$
\begin{aligned}
\left(d_{N} \alpha\right)\left(X_{0}, X_{1}, \cdots, X_{n}\right)=\sum_{i=0}^{n} & (-1)^{i}\left(N X_{i}\right) \alpha\left(X_{0}, \cdots, \widehat{X}_{i}, \cdots, X_{n}\right) \\
& +\sum_{i<j}(-1)^{i+j} \alpha\left(\left[X_{i}, X_{j}\right]_{N}, X_{0}, \cdots, \widehat{X}_{i}, \cdots, \widehat{X}_{j}, \cdots, X_{n}\right) .
\end{aligned}
$$

Moreover, as proved in [11], we have the following identity

$$
d_{N}=\left[i_{N}, d\right]=i_{N} d-d_{\circ} i_{N}
$$

The following proposition extends a result of Kosmann-Schwarzbach [11, Proposition 3.2].

Proposition 3.5. The quadruple $(M, \pi, N, \phi)$ is a Poisson quasi-Nijenhuis manifold if, and only if, $\left(\left(T^{*} M\right)_{\pi}, d_{N}, \phi\right)$ is a quasi Lie bialgebroid and $\phi$ is a closed 3 -form. 
This is an immediate consequence of Fact 3.4 and the following two lemmas.

Lemma 3.6 ([11, Proposition 3.2]). Assume that $\pi \in \mathfrak{X}^{2}(M)$ is a Poisson tensor and $N: T M \rightarrow T M$ a $(1,1)$-tensor on $M$. The differential $d_{N}$ is a derivation of the graded Lie algebra $\left(\Omega^{\bullet}(M),[\cdot, \cdot]_{\pi^{\sharp}}\right)$ if, and only if, $\pi$ and $N$ are compatible.

Lemma 3.7. Let $(M, \pi)$ be a Poisson manifold and $N: T M \rightarrow T M$ a $(1,1)$-tensor compatible with $\pi^{\sharp}$. Then $d_{N}^{2}=[\phi, \cdot]_{\pi^{\sharp}}$ if, and only if,

$$
[N X, N Y]-N([N X, Y]+[X, N Y]-N[X, Y])=\pi^{\sharp}\left(i_{X \wedge Y} \phi\right), \quad \forall X, Y \in \mathfrak{X}(M)
$$

and $\pi^{\#}{ }_{\circ}(d \phi)_{b}=0$, where $(d \phi)_{b}: \wedge^{3} T M \rightarrow T^{*} M$ is the bundle map defined by $(d \phi)_{b}(u, v, w)=$ $i_{u \wedge v \wedge w} d \phi, \forall u, v, w \in T M$.

Proof. It follows from an easy computation that

$$
\left(d_{N}^{2} f-[\phi, f]_{\pi^{\sharp}}\right)(X, Y)=(d f)\left([N X, N Y]-N([N X, Y]+[X, N Y]-N[X, Y])-\pi^{\sharp}\left(i_{X \wedge Y} \phi\right)\right)
$$

for all $f \in C^{\infty}(M)$. Moreover, since $d_{\circ} d_{N}+d_{N} \circ d=0$, one has

$$
d_{N}^{2}(d f)-[\phi, d f]_{\pi^{\sharp}}=d\left(d_{N}^{2} f\right)-\left(d[\phi, f]_{\pi^{\sharp}}-[d \phi, f]_{\pi^{\sharp}}\right)=d\left(d_{N}^{2} f-[\phi, f]_{\pi^{\sharp}}\right)+[d \phi, f]_{\pi^{\sharp}} .
$$

Hence, $d_{N}^{2}-[\phi, \cdot]_{\pi^{\sharp}}$ vanishes on 0 - and exact 1 -forms if, and only if,

$$
[N X, N Y]-N([N X, Y]+[X, N Y]-N[X, Y])=\pi^{\sharp}\left(i_{X \wedge Y} \phi\right), \quad \forall X, Y \in \mathfrak{X}(M)
$$

and $[d \phi, f]_{\pi^{\sharp}}=0, \forall f \in C^{\infty}(M)$. The latter is easily seen to be equivalent to $\pi^{\#}{ }_{\circ}(d \phi)_{b}=0$. And in this case, since both $d_{N}^{2}$ and $[\phi, \cdot]_{\pi^{\sharp}}$ are derivations with respect to $\wedge$, we get $d_{N}^{2}=[\phi, \cdot]_{\pi^{\sharp}}$.

As an immediate consequence, we obtain the following result of Kosmann-Schwarzbach [11].

Corollary 3.8. The triple $(M, \pi, N)$ is a Poisson Nijenhuis manifold if, and only if, $\left(\left(T^{*} M\right)_{\pi}, d_{N}\right)$ is a Lie bialgebroid.

We now turn our attention to the particular case where the Poisson bivector field $\pi$ is nondegenerate. Together with Lemma 3.6, the following two lemmas give another proof of the equivalence between the relation $\left[\pi, \pi_{N}\right]=0$ and the compatibility condition (7) when $\pi$ is non-degenerate (see Fact 3.2).

Lemma 3.9. Assume that $\pi \in \mathfrak{X}^{2}(M)$ is a Poisson tensor and $N: T M \rightarrow T M$ a $(1,1)$-tensor on $M$. Then $\pi_{N}$ is a bivector field such that $\left[\pi, \pi_{N}\right]=0$ if, and only if, all the squares in the following diagram commute.

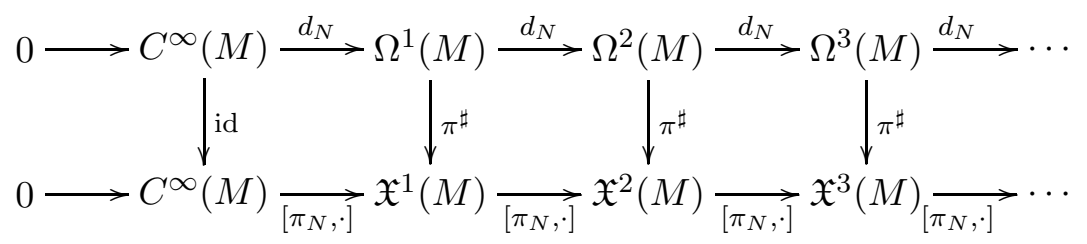

Proof. We have $\pi^{\sharp} N^{T}=N \pi^{\sharp}$ (i.e. $\pi_{N}$ is a bivector field) if, and only if, $\forall f \in C^{\infty}(M)$,

$$
\begin{array}{ll} 
& \pi^{\sharp} N^{T} d f=N \pi^{\sharp} d f \\
\Leftrightarrow & \pi^{\sharp} i_{N} d f=\pi_{N}^{\sharp} d f \\
\Leftrightarrow & \pi^{\sharp} d_{N} f=\left[\pi_{N}, f\right] .
\end{array}
$$


And $\left[\pi_{N}, \pi\right]=0$ is equivalent to

$$
\begin{array}{ll} 
& {\left[\pi_{N}, \pi\right]^{\sharp}(d f)=0} \\
\Leftrightarrow & {\left[\left[\pi_{N}, \pi\right], f\right]=0} \\
\Leftrightarrow & {\left[\left[\pi_{N}, f\right], \pi\right]+\left[\pi_{N},[\pi, f]\right]=0} \\
\Leftrightarrow & {\left[\pi_{N}^{\sharp} d f, \pi\right]+\left[\pi_{N}, \pi^{\sharp} d f\right]=0} \\
\Leftrightarrow & {\left[\pi, \pi^{\sharp} N^{T} d f\right]=\left[\pi_{N}, \pi^{\sharp} d f\right]} \\
\Leftrightarrow & {\left[\pi, \pi^{\sharp}\left(i_{N} d f\right)\right]=\left[\pi_{N}, \pi^{\sharp} d f\right]} \\
\Leftrightarrow & \pi^{\sharp} d\left(i_{N} d f\right)=\left[\pi_{N}, \pi^{\sharp} d f\right] \\
\Leftrightarrow & \pi^{\sharp} d_{N}(d f)=\left[\pi_{N}, \pi^{\sharp} d f\right]
\end{array}
$$

for all $f \in C^{\infty}(M)$. Since both $\pi^{\sharp} d_{N}$ and $\left[\pi_{N}, \pi^{\sharp}(\cdot)\right]$ are derivations of $\left(\Omega^{\bullet}(M)\right.$, $\left.\wedge\right)$, the equivalence follows from Eqs. (12)-(13).

Lemma 3.10. Assume that $\pi \in \mathfrak{X}^{2}(M)$ is a non-degenerate Poisson tensor, and $N: T M \rightarrow$ $T M$ is a $(1,1)$-tensor on $M$. If $\pi_{N}$ is a bivector field and Diagram (11) commutes, then $d_{N}$ is a derivation of $[\cdot, \cdot]_{\pi^{\sharp}}$.

Proof. Since $\pi$ is Poisson, we have

$$
\pi^{\sharp}[\alpha, \beta]_{\pi^{\sharp}}=\left[\pi^{\sharp} \alpha, \pi^{\sharp} \beta\right], \quad \forall \alpha, \beta \in \Omega^{\bullet}(M) .
$$

Then, the Jacobi identity for the Schouten bracket gives

$$
\left[\pi_{N}, \pi^{\sharp}[\alpha, \beta]_{\pi^{\sharp}}\right]=\left[\left[\pi_{N}, \pi^{\sharp} \alpha\right], \pi^{\sharp} \beta\right]+\left[\pi^{\sharp} \alpha,\left[\pi_{N}, \pi^{\sharp} \beta\right]\right],
$$

which can be rewritten as

$$
\pi^{\sharp} d_{N}\left([\alpha, \beta]_{\pi^{\sharp}}\right)=\pi^{\sharp}\left(\left[d_{N} \alpha, \beta\right]_{\pi^{\sharp}}+\left[\alpha, d_{N} \beta\right]_{\pi^{\sharp}}\right)
$$

since $\pi^{\sharp} d_{N}=\left[\pi_{N}, \pi^{\sharp}(\cdot)\right]$. The conclusion follows from the invertibility of $\pi^{\sharp}$.

The previous lemmas are used to prove the following

Proposition 3.11. (i) Let $(M, \pi, N, \phi)$ be a Poisson quasi-Nijenhuis manifold. Then,

$$
\left[\pi, \pi_{N}\right]=0
$$

and

$$
\left[\pi_{N}, \pi_{N}\right]=2 \pi^{\sharp}(\phi) .
$$

(ii) Conversely, assume that $\pi \in \mathfrak{X}^{2}(M)$ is a non-degenerate Poisson bivector field, $N$ : $T M \rightarrow T M$ is a $(1,1)$-tensor and $\phi$ is a closed 3-form. If Eqs. (14)-(15) are satisfied, then $(M, \pi, N, \phi)$ is a Poisson quasi-Nijenhuis manifold.

Proof. (i) Fact 3.2 implies Eq. (14). By Proposition 3.5, $\left(\left(T^{*} M\right)_{\pi}, d_{N}, \phi\right)$ is a quasi-Lie bialgebroid. It is simple to see that its induced bivector field on $M$ as in Proposition 4.8 of [9] is $\pi_{N}$. From Proposition 4.8 of [9], it follows that $\left[\pi_{N}, \pi_{N}\right]=2 \pi^{\sharp}(\phi)$. 
(ii) Since $\left[\pi, \pi_{N}\right]=0$, Lemma 3.9 implies that $\pi^{\sharp}{ }_{0} d_{N}=\left[\pi_{N}, \pi^{\sharp}(\cdot)\right]$ and Lemma 3.10 implies that $d_{N}$ is a derivation of $[\cdot, \cdot]_{\pi^{\sharp}}$. Hence $\pi$ and $N$ are compatible by Lemma 3.6. Since $\pi$ is non-degenerate, we may apply $\left(\pi^{\sharp}\right)^{-1}$ to Eq. (15). Then, making use of Lemma 3.9, we get back to $d_{N}^{2}=[\phi, \cdot]_{\pi^{\sharp}}$. Eq. (15) and the graded Jacobi identity yield $\left[\pi_{N}, \pi^{\sharp}(\phi)\right]=0$. Applying $\left(\pi^{\sharp}\right)^{-1}$, we get $d_{N} \phi=0$.

Corollary 3.12. Let $\omega$ be a symplectic 2 -form and $\phi$ a closed 3 -form on $M$. Then $(M, \omega, N, \phi)$ is a symplectic quasi-Nijenhuis manifold if and only if

$$
\left[\omega_{N}, \omega_{N}\right]=2 \phi \quad \text { and } \quad d \omega_{N}=0
$$

where $[\cdot, \cdot]$ stands for the Schouten bracket on $\Omega^{\bullet}(M)$ induced from the Lie algebroid $\left(T^{*} M\right)_{\pi}$, and $\omega_{N}$ is the 2 -form on $M$ defined by

$$
\omega_{N}(X, Y)=\omega(N X, Y), \quad \forall X, Y \in \mathfrak{X}(M) .
$$

Proof. It is well known that, when $\pi$ is non-degenerate, $\pi^{\sharp}$ is an isomorphism of differential Gerstenhaber algebras from $\left(\Omega^{\bullet}(M), d,[\cdot, \cdot]\right)$ to $\left(\mathfrak{X}^{\bullet}(M),[\pi, \cdot],[\cdot, \cdot]\right)[23,10]$. The conclusion thus follows immediately from Proposition 3.11 since $\pi^{\sharp} \omega_{N}=\pi_{N}$.

Remark 3.13. Poisson Nijenhuis structures arise naturally in the study of integrable systems. It would be interesting to find applications of Poisson quasi-Nijenhuis structures in integrable systems as well.

\section{Universal lifting theorem}

In this section, we recall the universal lifting theorem and its basic ingredients, as it plays a crucial role in the following sections. For details, see [9].

Let $\Gamma \rightrightarrows M$ be a Lie groupoid, $A \rightarrow M$ its Lie algebroid and $\Pi \in \mathfrak{X}^{k}(\Gamma)$ a $k$-vector field on $\Gamma$. Define $F_{\Pi} \in C^{\infty}\left(T^{*} \Gamma \times_{\Gamma} \stackrel{(k)}{\cdot} \times_{\Gamma} T^{*} \Gamma\right)$ by

$$
F_{\Pi}\left(\mu^{1}, \ldots, \mu^{k}\right)=\Pi\left(\mu^{1}, \ldots, \mu^{k}\right) .
$$

Definition 4.1. A $k$-vector field $\Pi \in \mathfrak{X}^{k}(\Gamma)$ is multiplicative if, and only if, $F_{\Pi}$ is a 1-cocycle with respect to the groupoid $T^{*} \Gamma \times_{\Gamma} \stackrel{(k)}{.} \times_{\Gamma} T^{*} \Gamma \rightrightarrows A^{*} \times_{M} \stackrel{(k)}{.} \times_{M} A^{*}$.

Remark 4.2. It is simple to see that a bivector field $\Pi$ is multiplicative if, and only if, the graph of the multiplication $\Lambda \subset \Gamma \times \Gamma \times \Gamma$ is coisotropic with respect to $\Pi \oplus \Pi \oplus \bar{\Pi}$, where $\bar{\Pi}$ denotes the opposite bivector field to $\Pi$.

Example 4.3. If $P \in \Gamma\left(\wedge^{k} A\right)$, then $\vec{P}-\overleftarrow{P}$ is multiplicative, where $\vec{P}$ and $\overleftarrow{P}$ denote, respectively, the right and left invariant $k$-vector fields on $\Gamma$ corresponding to $P$.

By $\mathfrak{X}_{\text {mult }}^{k}(\Gamma)$ we denote the space of all multiplicative k-vector fields on $\Gamma$. And $\mathfrak{X}_{\text {mult }}(\Gamma)=$ $\bigoplus_{k} \mathfrak{X}_{\text {mult }}^{k}(\Gamma)$.

Proposition 4.4 ([9]). The vector space $\mathfrak{X}_{\text {mult }}(\Gamma)$ is closed under the Schouten bracket, and therefore is a graded Lie algebra. 
It is simple to show that for any given $\Pi \in \mathfrak{X}_{\text {mult }}^{k}(\Gamma)$ and any $X \in \Gamma\left(\wedge^{i} A\right)$, the $(k+i-1)$-vector field $[\overleftarrow{X}, \Pi]$ is always left invariant. Define $\overleftarrow{\delta_{\Pi} X} \in \Gamma\left(\wedge^{(k+i-1)} A\right)$ by

$$
\overleftarrow{\delta_{\Pi} X}=[\overleftarrow{X}, \Pi]
$$

Thus one obtains a linear operator $\delta_{\Pi}: \Gamma\left(\wedge^{i} A\right) \rightarrow \Gamma\left(\wedge^{(k+i-1)} A\right)$. Here we use the following convention: $\Gamma\left(\wedge^{0} A\right) \cong C^{\infty}(M)$ and for any $f \in C^{\infty}(M), \overleftarrow{f}=\beta^{*} f$. One easily checks that the following identities are satisfied

$$
\begin{gathered}
\delta_{\Pi}(P \wedge Q)=\left(\delta_{\Pi} P\right) \wedge Q+(-1)^{p(k-1)} P \wedge \delta_{\Pi} Q, \\
\delta_{\Pi}[P, Q]=\left[\delta_{\Pi} P, Q\right]+(-1)^{(p-1)(k-1)}\left[P, \delta_{\Pi} Q\right],
\end{gathered}
$$

for all $P \in \Gamma\left(\wedge^{p} A\right)$ and $Q \in \Gamma\left(\wedge^{q} A\right)$. This leads to the following definition of $k$-differentials. Recall that for any Lie algebroid $A \rightarrow M,\left(\Gamma\left(\wedge^{\bullet} A\right), \wedge,[\cdot, \cdot]\right)$ is a Gerstenhaber algebra [23].

Definition 4.5. A k-differential on a Lie algebroid $A$ is a degree $(k-1)$ derivation of the Gerstenhaber algebra $\left(\Gamma\left(\wedge^{\bullet} A\right), \wedge,[\cdot, \cdot]\right)$. I.e. a linear operator

$$
\delta: \Gamma\left(\wedge^{\bullet} A\right) \rightarrow \Gamma\left(\wedge^{\bullet+(k-1)} A\right)
$$

satisfying

$$
\begin{gathered}
\delta(P \wedge Q)=(\delta P) \wedge Q+(-1)^{p(k-1)} P \wedge \delta Q, \\
\delta[P, Q]=[\delta P, Q]+(-1)^{(p-1)(k-1)}[P, \delta Q],
\end{gathered}
$$

for all $P \in \Gamma\left(\wedge^{p} A\right)$ and $Q \in \Gamma\left(\wedge^{q} A\right)$. The set of $k$-differentials on $A$ is denoted by $\mathcal{A}^{k}(A)$.

The space of all multi-differentials $\mathcal{A}(A)=\bigoplus_{k} \mathcal{A}^{k}(A)$ becomes a graded Lie algebra when endowed with the graded commutator:

$$
\left[\delta_{1}, \delta_{2}\right]=\delta_{1} \circ \delta_{2}-(-1)^{(k-1)(l-1)} \delta_{2} \circ \delta_{1}, \quad \text { where } \delta_{1} \in \mathcal{A}^{k}(A) \text { and } \delta_{2} \in \mathcal{A}^{l}(A) .
$$

Below is a list of basic examples.

Examples 4.6. (i) When $A$ is a Lie algebra $\mathfrak{g}$, then k-differentials are in one-one correspondence with Lie algebra 1-cocycles $\delta: \mathfrak{g} \rightarrow \wedge^{k} \mathfrak{g}$ with respect to the adjoint action.

(ii) The 0 -differentials correspond to sections $\phi \in \Gamma\left(A^{*}\right)$ such that $d_{A} \phi=0$, i.e. Lie algebroid 1-cocycles with trivial coefficients.

(iii) The 1-differentials correspond to the infinitesimals of Lie algebroid automorphisms.

(iv) If $P \in \Gamma\left(\wedge^{k} A\right)$, then $\operatorname{ad}_{P}=[P, \cdot]$ is clearly a $k$-differential, which is called the coboundary $k$-differential associated to $P$.

(v) A Lie bialgebroid can be seen as a Lie algebroid together with a 2-differential of square zero. The converse is also true.

$>$ From the previous discussion, we know that there exists a linear map

$$
\mathfrak{X}_{\text {mult }}^{\bullet}(\Gamma) \rightarrow \mathcal{A}^{\bullet}(A): \Pi \mapsto \delta_{\Pi},
$$

which is a Lie algebra homomorphism since the graded Jacobi identity satisfied by the Schouten bracket implies that

$$
\left[\delta_{\Pi}, \delta_{\Pi^{\prime}}\right]=\delta_{\left[\Pi, \Pi^{\prime}\right]}
$$

Moreover, one has the following 
Universal Lifting Theorem ([9]). Assume that $\Gamma \rightrightarrows M$ is a target-connected and targetsimply connected Lie groupoid with Lie algebroid A. Then

$$
\mathfrak{X}_{\text {mult }}^{\bullet}(\Gamma) \rightarrow \mathcal{A}^{\bullet}(A): \Pi \mapsto \delta_{\Pi}
$$

is an isomorphism of graded Lie algebras.

\section{$5 \quad$ Symplectic Nijenhuis groupoids}

Definition 5.1. A symplectic Nijenhuis groupoid is a symplectic groupoid $(\Gamma \rightrightarrows M, \widetilde{\omega})$ equipped with a multiplicative $(1,1)$-tensor $\widetilde{N}: T \Gamma \rightarrow T \Gamma$ such that $(\Gamma, \widetilde{\omega}, \widetilde{N})$ is a symplectic Nijenhuis structure.

The main result of this section is the following

Theorem 5.2. (i) The unit space of a symplectic Nijenhuis groupoid is a Poisson Nijenhuis manifold.

(ii) Every integrable Poisson Nijenhuis manifold is the unit space of a unique target-connected, target-simply connected symplectic Nijenhuis groupoid.

Here, by an integrable Poisson Nijenhuis manifold, we mean the corresponding Poisson structure is integrable, i.e. it admits an associated symplectic groupoid. See [5, 6] for the solution of the integrability problem for Poisson manifolds and, more generally, Lie algebroids.

Recall that a Poisson Nijenhuis manifold $(M, \pi, N)$ gives rise to a Lie bialgebroid $\left(\left(T^{*} M\right)_{\pi}, d_{N}\right)$ according to Corollary 3.8. The following lemma gives a useful characterization of those Lie bialgebroids arising from Poisson Nijenhuis structures.

Lemma 5.3. Let $(M, \pi)$ be a Poisson manifold. A Lie bialgebroid $\left(\left(T^{*} M\right)_{\pi}, \delta\right)$ is induced by a Poisson Nijenhuis structure if and only if $[\delta, d]=0$, where $d$ stands for the de Rham differential.

Proof. If $(M, \pi, N)$ is a Poisson Nijenhuis manifold, then $d_{N}=i_{N \circ} d-d_{\circ} i_{N}$. Thus

$$
\left[d_{N}, d\right]=d_{N} \circ d+d_{\circ} d_{N}=\left(i_{N} \circ d-d_{\circ} i_{N}\right) \circ d+d_{\circ}\left(i_{N} \circ d-d_{\circ} i_{N}\right)=0 .
$$

Conversely, given a Lie bialgebroid $\left(\left(T^{*} M\right)_{\pi}, \delta\right)$ such that $[\delta, d]=0$, one obtains a Lie algebroid structure on $T M$. Let $N: T M \rightarrow T M$ be its anchor map. Thus $\delta=d_{N}: C^{\infty}(M) \rightarrow \Omega^{1}(M)$. Since $[\delta, d]=0$, we have $\forall f \in C^{\infty}(M), \delta(d f)=-d \delta f=-d d_{N} f=d_{N}(d f)$. It thus follows that $\delta=d_{N}$ on any differential forms since both $\delta$ and $d_{N}$ are derivations and they agree on 0 and exact 1-forms. According to Corollary 3.8, it follows that $(M, \pi, N)$ is a Poisson Nijenhuis manifold.

Proof of Theorem 5.2. (i) From symplectic Nijenhuis groupoids to Poisson NijenHUIS MANIfOLDS. Assume that $(\Gamma, \widetilde{\omega}, \widetilde{N})$ is a symplectic Nijenhuis groupoid. Let $\widetilde{\pi}$ be the bivector field on $\Gamma$ which is the inverse of $\widetilde{\omega}$ and $\widetilde{\pi}_{\widetilde{N}} \in \mathfrak{X}^{2}(\Gamma)$ be the bivector field defined by $\widetilde{\pi}_{\widetilde{N}}^{\sharp}=\tilde{N}_{\circ} \widetilde{\pi}^{\sharp}$. 
- Since $[\tilde{\pi}, \tilde{\pi}]=0$, the induced bivector field $\pi=t_{*} \tilde{\pi}$ on the base manifold of the symplectic groupoid $\Gamma \rightrightarrows M$ is Poisson [22]. The Lie algebroid of $\Gamma \rightarrow M$ is isomorphic to $\left(T^{*} M\right)_{\pi}[2]$. And the multiplicative bivector field $\widetilde{\pi}$ corresponds to a 2-differential on $\left(T^{*} M\right)_{\pi}$, which is the de Rham differential $d$. That is, $\left(\left(T^{*} M\right)_{\pi}, d\right)$ is the Lie bialgebroid corresponding to the symplectic groupoid $(\Gamma, \widetilde{\omega})$.

- As pointed out in Fact $3.2, \widetilde{\pi}_{\widetilde{N}}$ is a Poisson tensor on $\Gamma[16,12,21]$. Moreover, $\tilde{\pi}_{\widetilde{N}}$ is a multiplicative bivector field since $\widetilde{N}$ is a multiplicative $(1,1)$-tensor and $\widetilde{\pi}$ is a multiplicative bivector field. In other words, $\left(\Gamma, \widetilde{\pi}_{\tilde{N}}\right)$ is a Poisson groupoid [14]. Let $\delta_{\widetilde{\pi}_{\tilde{N}}}: \Omega^{\bullet}(M) \rightarrow \Omega^{\bullet+1}(M)$ be the 2-differential on $\left(T^{*} M\right)_{\pi}$ induced by the multiplicative Poisson bivector field $\widetilde{\pi}_{\tilde{N}}$ on $\Gamma$. Since $\left[\widetilde{\pi}_{\widetilde{N}}, \widetilde{\pi}_{\tilde{N}}\right]=0$, the universal lifting theorem implies that

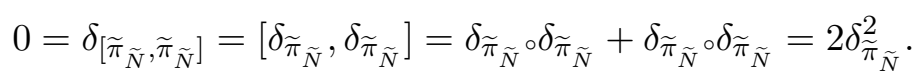

Thus, $\left(\left(T^{*} M\right)_{\pi}, \delta_{\widetilde{\pi}_{\tilde{N}}}\right)$ is a Lie bialgebroid.

- Likewise, it is standard that $\left[\widetilde{\pi}_{\tilde{N}}, \tilde{\pi}\right]=0$. Thus the universal lifting theorem implies that $\left[\delta_{\tilde{\pi}_{\tilde{N}}}, d\right]=0$. According to Lemma $5.3, \delta_{\tilde{\pi}_{\tilde{N}}}=d_{N}$ for some Nijenhuis tensor $N$ on $M$ and $(M, \pi, N)$ is a Poisson Nijenhuis manifold.

(ii) From Poisson Nijenhuis manifolds to symplectic Nijenhuis groupoids. Given a Poisson Nijenhuis manifold $(M, \pi, N)$, then $\left(\left(T^{*} M\right)_{\pi}, d_{N}\right)$ is a Lie bialgebroid by Corollary 3.8. Assume that $\left(T^{*} M\right)_{\pi}$ is integrable (see $[5,6]$ for the integrability condition) and $(\Gamma \rightrightarrows M, \widetilde{\omega})$ is a target-connected and target simply-connected symplectic groupoid of $M$. Since $d_{N}^{2}=0$ and $\left[d_{N}, d\right]=0$, the universal lifting theorem implies that $d_{N}$ corresponds to a multiplicative Poisson bivector field $\widetilde{\pi}_{\tilde{N}}$ on $\Gamma$ such that $\left[\widetilde{\pi}_{\tilde{N}}, \widetilde{\pi}\right]=0$, where $\tilde{\pi}$ is the Poisson tensor on $\Gamma$ inverse to $\widetilde{\omega}$. Let $\widetilde{N}=\widetilde{\pi}_{\widetilde{N}}^{\sharp} \circ \widetilde{\omega}_{b}: T \Gamma \rightarrow T \Gamma$. Then it is clear that $\widetilde{N}$ is a multiplicative $(1,1)$-tensor, and $(\Gamma, \widetilde{\omega}, \widetilde{N})$ is a symplectic Nijenhuis groupoid.

Since these two constructions are inverse to each other, the theorem is proved.

\section{Symplectic quasi-Nijenhuis groupoids}

The goal of this section is to generalize Theorem 5.2 to the quasi-setting. More precisely, we will give an integration theorem for Poisson quasi-Nijenhuis manifolds.

Definition 6.1. A symplectic quasi-Nijenhuis groupoid is a symplectic groupoid $(\Gamma \rightrightarrows M, \widetilde{\omega})$ equipped with a multiplicative $(1,1)$-tensor $\widetilde{N}: T \Gamma \rightarrow T \Gamma$ and a closed 3-form $\phi \in \Omega^{3}(M)$ such that $\left(\Gamma, \widetilde{\omega}, \widetilde{N}, t^{*} \phi-s^{*} \phi\right)$ is a symplectic quasi-Nijenhuis structure.

The following result is a generalization of Theorem 5.2.

Theorem 6.2. (i) The unit space of a symplectic quasi-Nijenhuis groupoid is a Poisson quasi-Nijenhuis manifold.

(ii) Every integrable Poisson quasi-Nijenhuis manifold $(M, \pi, N, \phi)$ is the unit space of a unique target-connected and target-simply connected symplectic quasi-Nijenhuis groupoid $\left(\Gamma \rightrightarrows M, \widetilde{\omega}, \widetilde{N}, t^{*} \phi-s^{*} \phi\right)$.

Proof. The proof is similar to that of Theorem 5.2, so we will merely sketch it. 
Assume that $(M, \pi, N, \phi)$ is an integrable Poisson quasi-Nijenhuis manifold. Let $\Gamma \rightrightarrows M$ be a target-connected and target-simply connected groupoid integrating the Lie algebroid $\left(T^{*} M\right)_{\pi}$. By Proposition 3.5, $\left(\left(T^{*} M\right)_{\pi}, d_{N}, \phi\right)$ is a quasi-Lie bialgebroid, which integrates to a quasiPoisson groupoid by the universal lifting theorem. Let $\widetilde{\pi}_{\tilde{N}} \in \mathfrak{X}(\Gamma)$ be the bivector field on $\Gamma$ corresponding to $d_{N}$. Then we have

$$
\frac{1}{2}\left[\widetilde{\pi}_{\widetilde{N}}, \widetilde{\pi}_{\widetilde{N}}\right]=\vec{\phi}-\overleftarrow{\phi}
$$

On the other hand, we know that $\Gamma \rightrightarrows M$ is a symplectic groupoid, whose corresponding Lie bialgebroid is $\left(\left(T^{*} M\right)_{\pi}, d\right)$. The symplectic form on $\Gamma$ is denoted by $\widetilde{\omega}$. Let $\widetilde{\pi} \in \mathfrak{X}^{2}(\Gamma)$ be its corresponding Poisson tensor. Since $\left[d_{N}, d\right]=0$, we have $\left[\widetilde{\pi}_{\tilde{N}}, \widetilde{\pi}\right]=0$ according to the universal lifting theorem. Let $\widetilde{N}=\widetilde{\pi}_{\widetilde{N}}^{\sharp} \widetilde{\omega}_{b}: T \Gamma \rightarrow T \Gamma$. Then it is clear that $\widetilde{N}$ is a multiplicative $(1,1)-$ tensor. Since $\vec{\phi}-\overleftarrow{\phi}=\widetilde{\pi}^{\sharp}\left(t^{*} \phi-s^{*} \phi\right)$, from Proposition 3.11, it follows that $\left(\Gamma, \widetilde{\omega}, \widetilde{N}, t^{*} \phi-s^{*} \phi\right)$ is a symplectic quasi-Nijenhuis groupoid.

The other direction can be proved by going backwards.

Remark 6.3. Note that $\widetilde{\omega}_{b}\left(\widetilde{\pi}_{\widetilde{N}}\right)$ is a multiplicative 2 -form on $\Gamma \rightrightarrows M$. It would be interesting to see what is the corresponding Dirac structure on $M$ and how the integration result in [1] can be applied to this situation.

\section{Generalized complex structures}

This section is devoted to the investigation of the relationship between generalized complex structures and Poisson quasi-Nijenhuis structures. Let us first recall the definition of generalized complex structures $[8,7]$.

Definition 7.1. A generalized complex structure on a manifold $M$ is a bundle map

$$
J: T M \oplus T^{*} M \rightarrow T M \oplus T^{*} M
$$

satisfying the algebraic properties

$$
J^{2}=-I \quad \text { and } \quad\langle J v, J w\rangle=\langle v, w\rangle
$$

and the integrability condition

$$
(J v, J w)-(v, w)-J((J v, w)+(v, J w))=0
$$

$\forall v, w \in \Gamma\left(T M \oplus T^{*} M\right)$. Here $\langle\cdot, \cdot\rangle$ and $(\cdot, \cdot)$ are the pairing and bracket on the standard Courant algebroid $T M \oplus T^{*} M$ as in Example 2.2.

The first two algebraic conditions (17) imply that $J$ must be of the form

$$
J=\left(\begin{array}{cc}
N & \pi^{\sharp} \\
\sigma_{b} & -N^{*}
\end{array}\right)
$$

where $\pi \in \mathfrak{X}^{2}(M)$ is a bivector field, $\sigma \in \Omega^{2}(M)$ is a 2-form and $N: T M \rightarrow T M$ is a $(1,1)$ tensor. Here $\sigma_{b}: T M \rightarrow T^{*} M$ is the map given by $\left(\sigma_{b} X\right)(Y)=\sigma(X, Y), \forall X, Y \in \mathfrak{X}(M)$. 
On the other hand, a Courant algebroid can be deformed using a bundle map $J$. More precisely, let $(E,\langle\cdot, \cdot\rangle, \llbracket \cdot, \cdot \rrbracket, \rho)$ be a Courant algebroid over $M$ and let

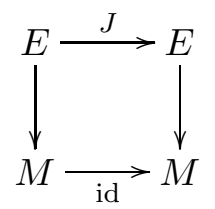

be a vector bundle automorphism of $E \rightarrow M$. Consider

- the inner product

$$
\langle A, B\rangle_{J}=\langle J A, J B\rangle
$$

- the bracket

$$
\llbracket A, B \rrbracket J=\llbracket J A, B \rrbracket+\llbracket A, J B \rrbracket-J \llbracket A, B \rrbracket
$$

- and the bundle map

$$
\rho_{J}=\rho_{\circ} J
$$

induced by $J$.

A natural question is

Question 7.2. When is the quadruple $\left(E,\langle\cdot, \cdot\rangle_{J}, \llbracket \cdot, \cdot \rrbracket_{J}, \rho_{J}\right)$ still a Courant algebroid?

The next proposition gives a trivial sufficient condition.

Proposition 7.3. The quadruple $\left(E,\langle\cdot, \cdot\rangle_{J}, \llbracket \cdot, \cdot \rrbracket_{J}, \rho_{J}\right)$ is a Courant algebroid if

$$
\llbracket J A, J B \rrbracket+J^{2} \llbracket A, B \rrbracket-J(\llbracket J A, B \rrbracket+\llbracket A, J B \rrbracket)=0, \quad \forall A, B \in \Gamma(E) .
$$

Moreover, in this case, $J$ is a Courant algebroid isomorphism from $\left(E,\langle\cdot, \cdot\rangle_{J}, \llbracket \cdot, \cdot \rrbracket_{J}, \rho_{J}\right)$ to $(E,\langle\cdot, \cdot\rangle, \llbracket \cdot, \cdot \rrbracket, \rho)$.

We now give an answer to Question 7.2 in the special case of the standard Courant algebroid $T M \oplus T^{*} M$, where $J$ satisfies Eqs. (17), and is given by Eq. (18) .

Lemma 7.4. Assume that $J: T M \oplus T^{*} M \rightarrow T M \oplus T^{*} M$ is given by Eq. (18). Let $\left(\cdot, \cdot D_{J}\right.$ be the deformed bracket on $\mathfrak{X}(M) \oplus \Omega^{1}(M)$ as in Eq. (19). Then, for all $X, Y \in \mathfrak{X}(M)$ and $\xi, \eta \in \Omega^{1}(M)$, we have

$$
\begin{gathered}
(\xi, \eta)_{J}=[\xi, \eta]_{\pi^{\sharp}} \\
(X, Y)_{J}=[X, Y]_{N}+(d \sigma)(X, Y, \cdot) \\
(X, \xi)_{J}=\left(\left[X, \pi^{\sharp} \xi\right]-\pi^{\sharp}\left(\mathcal{L}_{X} \xi-\frac{1}{2} d(\xi X)\right)\right)+\left(\mathcal{L}_{N X} \xi-\mathcal{L}_{X}\left(N^{T} \xi\right)+N^{T}\left(\mathcal{L}_{X} \xi-\frac{1}{2} d(\xi X)\right)\right)
\end{gathered}
$$

Proof. This follows from a straightforward computation using Eqs. (2) and (19), and is left for the reader.

Proposition 7.5. Let $J: T M \oplus T^{*} M \rightarrow T M \oplus T^{*} M$ be a bundle map which satisfies Eqs. (17), and is given by Eq. (18). Then $\left(T M \oplus T^{*} M,\langle\cdot, \cdot\rangle_{J},(\cdot, \cdot)_{J}, \rho_{J}\right)$ is a Courant algebroid if, and only if, $(M, \pi, N, d \sigma)$ is a Poisson quasi-Nijenhuis manifold. And in this case, $\left(T M \oplus T^{*} M,\langle\cdot, \cdot\rangle_{J},(\cdot, \cdot)_{J}, \rho_{J}\right)$ is naturally identified with the double of the quasi-Lie bialgebroid $\left(\left(T^{*} M\right)_{\pi}, d_{N}, d \sigma\right)$. 
Proof. Assume that $\left(T M \oplus T^{*} M,\langle\cdot, \cdot\rangle_{J},(\cdot, \cdot)_{J}, \rho_{J}\right)$ is a Courant algebroid. It is clear that $A:=$ $T^{*} M$ and $B:=T M$ are transversal, maximal isotropic subbundles. By Eq. (20), $A=T^{*} M$ is a Dirac structure with the induced bracket $[\cdot, \cdot]_{\pi^{\sharp}}$. Thus, according to Theorem 2.6, we obtain a quasi-Lie bialgebroid. The construction of the corresponding derivation $\delta$ of $\left(\Omega^{\bullet}(M), \wedge,[\cdot, \cdot]_{\pi^{\sharp}}\right)$ and twisting 3 -form $\phi$ was outlined in the proof of Theorem 2.6. In the present situation, we have

$$
\rho_{B}(X)=\rho_{J}(X)=\rho(J X)=\rho\left(N X+\sigma_{b} X\right)=N X, \quad \forall X \in T M
$$

and, combining Eqs. (21) and (6),

$$
[X, Y]_{B}=[X, Y]_{N}, \quad \forall X, Y \in \mathfrak{X}(M) .
$$

Therefore, comparing Eqs. (3) and (9), we conclude that $\delta=d_{N}$. And, combining Eqs. (5) and (21), we get

$$
\begin{aligned}
\phi(X, Y, Z)=2\left\langle(X, Y\rangle_{J}, Z\right\rangle_{J}=2\left\langle J(X, Y\rangle_{J}, J Z\right\rangle=2\left\langle\langle X, Y\rangle_{J}, Z\right\rangle \\
=2\left\langle[X, Y]_{N}+d \sigma(X, Y, \cdot), Z\right\rangle=d \sigma(X, Y, Z), \quad \forall X, Y, Z \in \mathfrak{X}(M) .
\end{aligned}
$$

Hence $\left(\left(T^{*} M\right)_{\pi}, d_{N}, d \sigma\right)$ is a quasi-Lie bialgebroid or, equivalently according to Proposition $3.5,(M, \pi, N, d \sigma)$ is a Poisson quasi-Nijenhuis manifold.

Conversely, assume that $(M, \pi, N, d \sigma)$ is a Poisson quasi-Nijenhuis manifold. By Proposition $3.5,\left(\left(T^{*} M\right)_{\pi}, d_{N}, d \sigma\right)$ is a quasi-Lie bialgebroid. Its double $E$ is a Courant algebroid. We will show that $E$ is indeed isomorphic to $\left(T M \oplus T^{*} M,\langle\cdot, \cdot\rangle_{J},(\cdot, \cdot)_{J}, \rho_{J}\right)$. First, it is simple to check that their anchors and non-degenerate symmetric pairings coincide. It remains to check that their brackets coincide. According to Eq. (4), the bracket $\llbracket \cdot, \cdot \rrbracket$ on $\Gamma(E)$ is given by

$$
\begin{gathered}
\llbracket \xi, \eta \rrbracket=[\xi, \eta]_{\pi} \\
\llbracket X, Y \rrbracket=[X, Y]_{N}+(d \sigma)(X, Y, \cdot) \\
\llbracket X, \xi \rrbracket=\left(i_{X} \delta_{T M} \xi+\frac{1}{2} \delta_{T M}(\xi X)\right)-\left(i_{\xi} \delta_{T^{*} M} X+\frac{1}{2} \delta_{T^{*} M}(\xi X)\right)
\end{gathered}
$$

for all $X, Y \in \mathfrak{X}(M)$ and $\xi, \eta \in \Omega^{1}(M)$. In our case, we have

$$
\delta_{T^{*} M}=[\pi, \cdot] \quad \text { and } \quad \delta_{T M}=d_{N} .
$$

It follows from a straightforward verification that the right hand sides of Eqs. (20)-(22) and (23)-(25) coincide. Therefore, $\left(T M \oplus T^{*} M,\langle\cdot, \cdot\rangle_{J},(\cdot, \cdot)_{J}, \rho_{J}\right)$ is indeed a Courant algebroid.

We are now ready to state the main result of this section.

Theorem 7.6. Assume that $J: T M \oplus T^{*} M \rightarrow T M \oplus T^{*} M$ as given by Eq. (18) satisfies Eqs (17). Then the following are equivalent

- $J$ is a generalized complex structure;

- $(M, \pi, N, d \sigma)$ is a Poisson quasi-Nijenhuis manifold such that

$$
(T M)_{N} \oplus\left(T^{*} M\right)_{\pi} \stackrel{J}{\rightarrow} T M \oplus T^{*} M
$$

is a Courant algebroid isomorphism.

Here $(T M)_{N} \oplus\left(T^{*} M\right)_{\pi}$ denotes the Courant algebroid corresponding to the quasi-Lie bialgebroid $\left(\left(T^{*} M\right)_{\pi}, d_{N}, d \sigma\right)$. 


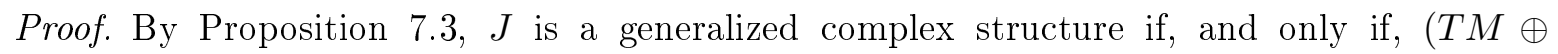
$\left.T^{*} M,\langle\cdot, \cdot\rangle_{J},(\cdot, \cdot)_{J}, \rho_{J}\right)$ is a Courant algebroid and $\left(T M \oplus T^{*} M,\langle\cdot, \cdot\rangle_{J}, \ell, \emptyset_{J}, \rho_{J}\right) \stackrel{J}{\rightarrow}(T M \oplus$ $\left.T^{*} M,\langle\cdot, \cdot\rangle,(\cdot, \cdot \cdot), \rho\right)$ is a Courant algebroid isomorphism. The result follows immediately from Proposition 7.5.

Since any generalized complex structure naturally gives rise to a Poisson quasi-Nijenhuis manifold, as an immediate consequence of Theorem 6.2 , we have the following

Theorem 7.7. Let $J$ be a generalized complex structure as given by $E q .(18)$, and $(\Gamma \rightrightarrows M, \widetilde{\omega})$ a target-connected and target-simply connected symplectic groupoid integrating $\left(T^{*} M\right)_{\pi}$. Then there is a multiplicative $(1,1)$-tensor $\widetilde{N}$ on $\Gamma$ such that $\left(\Gamma \rightrightarrows M, \widetilde{\omega}, \widetilde{N}, t^{*} d \sigma-s^{*} d \sigma\right)$ is a symplectic quasi-Nijenhuis groupoid.

Remark 7.8. Note that Theorem 3.3-3.4 in [4] essentially imply our Theorem 7.7. Our proof is conceptual, while Crainic used a direct argument. It would be interesting to see how Theorem 3.4 (ii) in [4] can be proved conceptually.

\section{References}

[1] H. Bursztyn, M. Crainic, A. Weinstein, and C. Zhu, Integration of twisted Dirac brackets, Duke Math. J. 123 (2004), no. 3, 549-607.

[2] A. Coste, P. Dazord, and A. Weinstein, Groupoïdes symplectiques, Publications du Département de Mathématiques. Nouvelle Série. A, Vol. 2, Publ. Dép. Math. Nouvelle Sér. A, 87, Univ. Claude-Bernard, Lyon, 1987, pp. i-ii, 1-62.

[3] T. J. Courant, Dirac manifolds, Trans. Amer. Math. Soc. 319 (1990), no. 2, 631-661.

[4] M. Crainic, Generalized complex structures and Lie brackets, arXiv:math.DG/0412097.

[5] M. Crainic, and R. L. Fernandes, Integrability of Lie brackets, Ann. of Math. (2) 157 (2003), no. 2, 575-620.

[6] _ Integrability of Poisson brackets, J. Differential Geom. 66 (2004), no. 1, 71-137.

[7] M. Gualtieri, Generalized complex geometry, arXiv:math.DG/0401221.

[8] N. Hitchin, Generalized Calabi-Yau manifolds, Q. J. Math. 54 (2003), no. 3, 281-308.

[9] D. Iglesias, C. Laurent-Gengoux, and P. Xu, Universal lifting theorem and quasi-Poisson groupoids, arXiv:math.DG/0507396.

[10] Y. Kosmann-Schwarzbach, Exact Gerstenhaber algebras and Lie bialgebroids, Acta Appl. Math. 41 (1995), no. 1-3, 153-165.

[11] _ The Lie bialgebroid of a Poisson-Nijenhuis manifold, Lett. Math. Phys. 38 (1996), no. $4,421-428$.

[12] Y. Kosmann-Schwarzbach and F. Magri, Poisson-Nijenhuis structures, Ann. Inst. H. Poincaré Phys. Théor. 53 (1990), no. 1, 35-81.

[13] Z.-J. Liu, A. Weinstein, and P. Xu, Manin triples for Lie bialgebroids, J. Differential Geom. 45 (1997), no. 3, 547-574. 
[14] K. C. H. Mackenzie and P. Xu, Lie bialgebroids and Poisson groupoids, Duke Math. J. 73 (1994), no. 2, 415-452.

[15] _ Integration of Lie bialgebroids, Topology 39 (2000), no. 3, 445-467.

[16] F. Magri and C. Morosi, On the reduction theory of the Nijenhuis operators and its applications to Gel'fand-Dikiu equations, Proceedings of the IUTAM-ISIMM symposium on modern developments in analytical mechanics, Vol. II (Torino, 1982), 117, 1983, pp. 599626.

[17] _ Old and new results on recursion operators: an algebraic approach to KP equation, Topics in soliton theory and exactly solvable nonlinear equations (Oberwolfach, 1986), World Sci. Publishing, Singapore, 1987, pp. 78-96.

[18] F. Magri, C. Morosi, and O. Ragnisco, Reduction techniques for infinite-dimensional Hamiltonian systems: some ideas and applications, Comm. Math. Phys. 99 (1985), no. 1, $115-140$.

[19] D. Roytenberg, Courant algebroids, derived brackets and even symplectic supermanifolds, arXiv:math.DG/9910078.

[20] _ Quasi-Lie bialgebroids and twisted Poisson manifolds, Lett. Math. Phys. 61 (2002), no. 2, 123-137.

[21] I. Vaisman, Complementary 2-forms of Poisson structures, Compositio Math. 101 (1996), no. $1,55-75$.

[22] A. Weinstein, Symplectic groupoids and Poisson manifolds, Bull. Amer. Math. Soc. (N.S.) 16 (1987), no. 1, 101-104.

[23] P. Xu, Gerstenhaber algebras and BV-algebras in Poisson geometry, Comm. Math. Phys. 200 (1999), no. 3, 545-560. 